\title{
3D-Printed Nanocellulose-Based Cushioning-Antibacterial Dual-Function Food Packaging Aerogel
}

\author{
Wei Zhou ${ }^{\dagger}$, Jiawei Fang ${ }^{\dagger}$, Shuwei Tang, Zhengguo Wu * and Xiaoying Wang (D) \\ State Key Laboratory of Pulp and Paper Engineering, South China University of Technology, 381 Wushan Road, \\ Tianhe District, Guangzhou 510640, China; annzhouw@outlook.com (W.Z.); fangjw010@163.com (J.F.); \\ T13061427625@163.com (S.T.); xyw@scut.edu.cn (X.W.) \\ * Correspondence: wuzhg@scut.edu.cn \\ t These two authors contributed equally to this work.
}

Citation: Zhou, W.; Fang, J.; Tang, S.; Wu, Z.; Wang, X. 3D-Printed

Nanocellulose-Based CushioningAntibacterial Dual-Function Food Packaging Aerogel. Molecules 2021, 26,3543. https://doi.org/10.3390/ molecules 26123543

Academic Editor: Alejandro Rodríguez Pascual

Received: 18 May 2021

Accepted: 7 June 2021

Published: 10 June 2021

Publisher's Note: MDPI stays neutral with regard to jurisdictional claims in published maps and institutional affiliations.

Copyright: (c) 2021 by the authors. Licensee MDPI, Basel, Switzerland. This article is an open access article distributed under the terms and conditions of the Creative Commons Attribution (CC BY) license (https:// creativecommons.org/licenses/by/ $4.0 /)$.

\begin{abstract}
Cushioning and antibacterial packaging are the requirements of the storage and transportation of fruits and vegetables, which are essential for reducing the irreversible quality loss during the process. Herein, the composite of carboxymethyl nanocellulose, glycerin, and acrylamide derivatives acted as the shell and chitosan/AgNPs were immobilized in the core by using coaxial 3D-printing technology. Thus, the 3D-printed cushioning-antibacterial dual-function packaging aerogel with a shell-core structure (CNGA/C-AgNPs) was obtained. The CNGA/C-AgNPs packaging aerogel had good cushioning and resilience performance, and the average compression resilience rate was more than 90\%. Although AgNPs was slowly released, CNGA/C-AgNPs packaging aerogel had an obvious antibacterial effect on E. coli and S. aureus. Moreover, the CNGA/C-AgNPs packaging aerogel was biodegradable. Due to the customization capabilities of 3D-printing technology, the prepared packaging aerogel can be adapted to more application scenarios by accurately designing and regulating the microstructure of aerogels, which provides a new idea for the development of food intelligent packaging.
\end{abstract}

Keywords: 3D printing; carboxymethyl nanocellulose; silver nanoparticles; cushioning; antibacterial

\section{Introduction}

During the transportation, fruits and vegetables are easily damaged by mechanical injury due to static pressure, shock, vibration and collision, which lead to irreversible quality loss [1,2]. According to statistics, the loss rate of fruits caused by mechanical damage in the transportation process is as high as 30\% [3]. However, the commonly used foamed polystyrene (EPS) and polyethylene (EPE) materials are hard to be degraded and recycled [4]. Therefore, it is urgent to develop an environment-friendly biodegradable cushioning packaging material for fruits and vegetables. Nanocellulose possesses the advantages of being biodegradable, renewable, safe, and non-toxic, and the high aspect ratio gives it better strength and toughness, which can be used as the matrix of cushioning packaging materials [5-7].

In the process of storage and transportation of fruits and vegetables, in addition to shock absorption and cushioning, there is another huge demand for antibacterial packaging [8]. Traditional cushioning packaging has almost no antibacterial activity, while the cushioning packaging with antibacterial function can effectively control the quality degradation of fruits and vegetables caused by the growth and reproduction of epidermal microorganisms after harvest, synergistically reducing the loss rate of fruits and vegetables [9]. Silver nanoparticles (AgNPs), a kind of broad-spectrum antibacterial agent, have been widely used in many fields, including food packaging [10-12], which could be used as a functional component to endow the cushioning food packaging materials with antibacterial activity. However, it is worth noting that there is a cumulative toxicity risk of silver 
nanoparticles, and its release needs to be controlled for reducing the potential harm to the human body and ecology during the applications $[13,14]$.

Coaxial 3D-printing technology has the ability to precisely design and construct fine structures of materials, which can be used to fabricate the shell-core structure for the immobilization of AgNPs in one step. More importantly, the customization capabilities of coaxial 3D-printing technology can accurately design and regulate the microstructure of materials according to different application scenarios [15], so as to obtain the most suitable customized structure and the best shock absorption and cushioning performance of the packaging material. Meanwhile, the highly programmed characteristics endow 3D-printed antibacterial and cushioning dual-function food packaging materials with the potential of large-scale industrialization.

Therefore, as shown in Figure 1, carboxymethyl nanocellulose (CMC) is used as the matrix, which is supplemented with acrylamide derivatives and glycerol to obtain CMCbased 3D-printing ink. With that ink as the shell material, chitosan/sliver nanoparticles (Cts/AgNPs) as the core layer, the biodegradable carboxymethyl nanocellulose-based antibacterial and cushioning dual functional food packaging aerogels were prepared by coaxial 3D-printing technology.

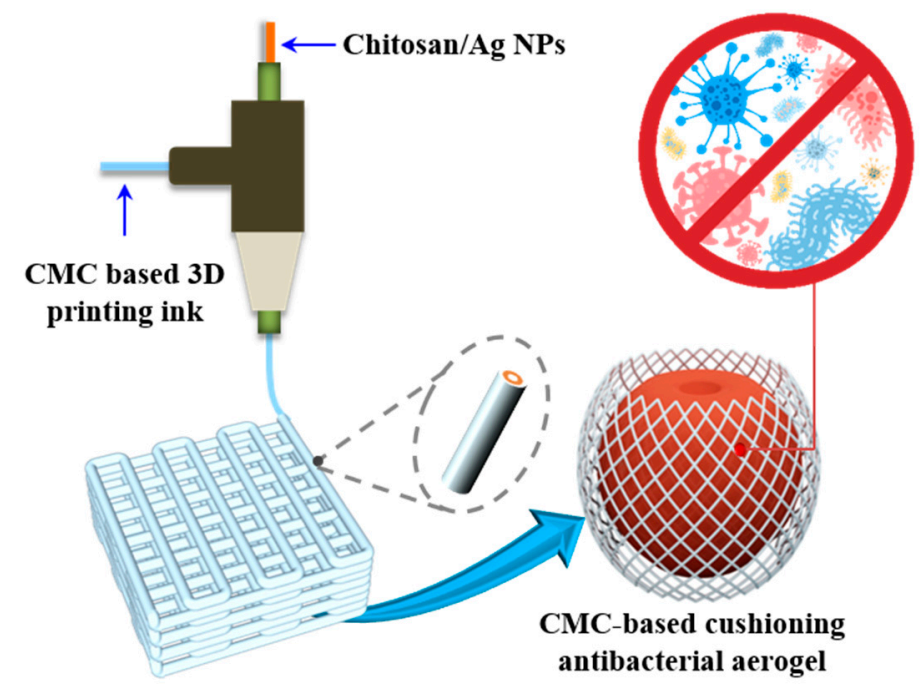

Figure 1. Scheme for the preparation process of 3D-printed carboxymethyl nanocellulose-based cushioning and antibacterial dual-function food packaging aerogels.

\section{Results and Discussion}

\subsection{Rheological Performances of 3D-Printing Inks}

The microscopic morphology of carboxymethyl nanocellulose (CMC) was characterized by SEM, and the results are shown in Figure 2a,b. The diameter of CMC was in the nanoscale range with a large aspect ratio. After freeze drying, the fibers were entangled to form a three-dimensional network structure, indicating that $\mathrm{CMC}$ is suitable for the construction of cushioning packaging aerogels.

Rheological performance is a key factor affecting the printability and fidelity of 3Dprinting inks. In this study, the rheological performances of CMC and CMC-based 3Dprinting inks were analyzed. The results are shown in Figure 2c,d. As can be seen from Figure 2c, CMC-based 3D-printing inks retained the shear thinning behavior of CMC. When the shear rate increased from $10^{-2} \mathrm{~s}^{-1}$ to $10^{2} \mathrm{~s}^{-1}$, the viscosity of CMC and CMC-based 3D-printing inks generally decreased by three orders of magnitude. The shear thinning behavior of CMC-based 3D-printing inks could reduce the extrusion pressure needed in the printing process, so that the inks could be extruded more smoothly from the needle, especially the coaxial needle. As can be seen from Figure 2c, with the increase of glycerol concentration in the ink, its viscosity gradually increased. The viscosity of 75Ink was up to 
26,854.14 $\mathrm{Pa}$ at $10^{-2} \mathrm{~s}^{-1}$. When the shear force was removed, CMC-based 3D-printing inks had a higher viscosity at low shear rates, which helped to maintain the shape after printing. As can be seen from Figure 2d, with the increase of glycerin content, the storage modulus, loss modulus, and yield stress of CMC-based 3D-printing inks were all improved, which promoted the ink to have higher printing fidelity in the 3D-printing process. In addition, the yield stress of 75Ink was the largest, reaching 275.30 Pa.

a
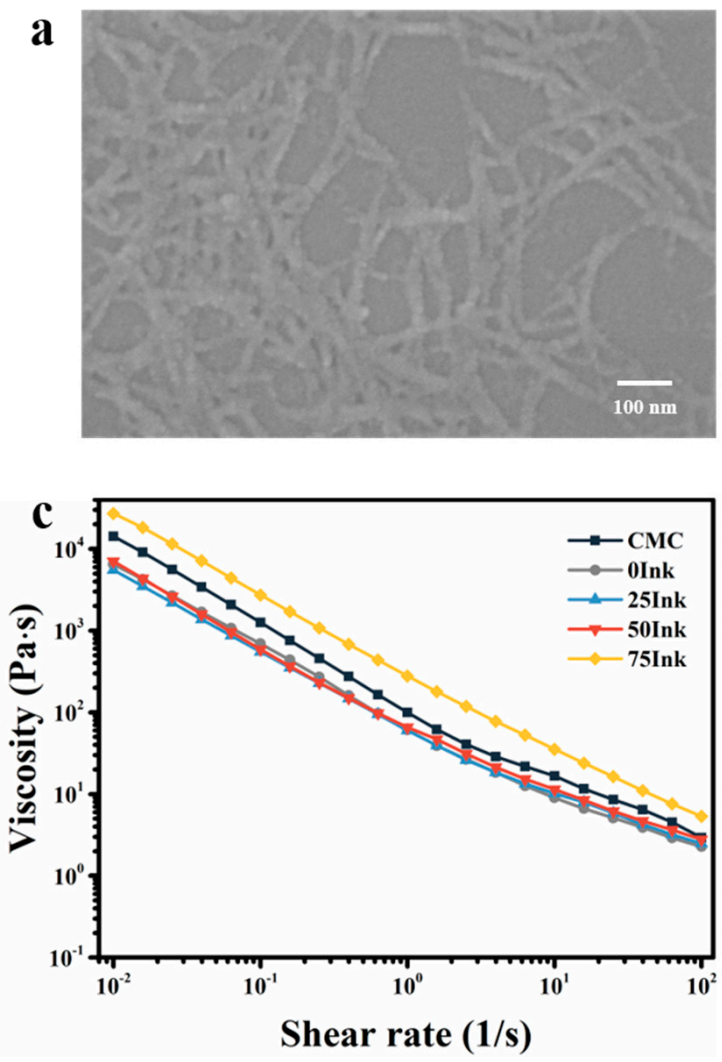
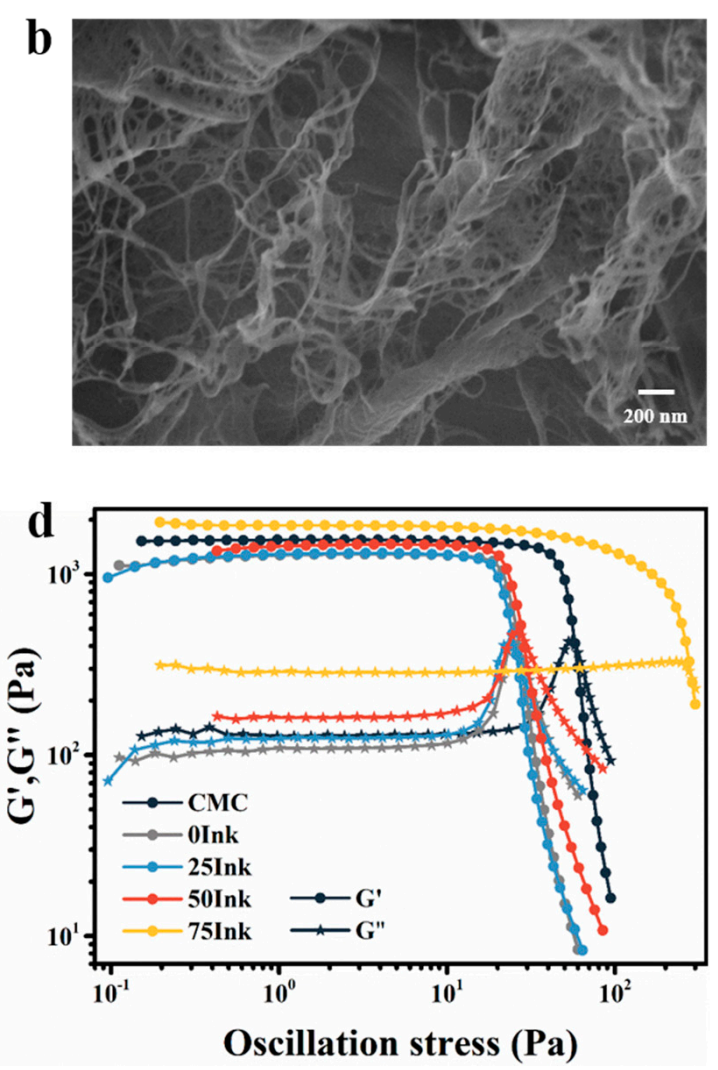

Figure 2. SEM images of CMC (a) before and (b) after freeze drying. (c) Viscosity as a function of the shear rate for CMC and CMC-based inks, (d) $G^{\prime}$ and $G^{\prime \prime}$ as a function of the shear stress for CMC and CMC-based inks.

\subsection{Effect of Glycerol Content on 3D-Printed Cushioning Aerogels}

The morphology and microstructure of CMC-based 3D-printed cushioning aerogels prepared by uniaxial 3D printing were preliminarily analyzed. As shown in Figure 3, CMCbased inks with $25 \mathrm{vol} \%$ (25Ink), $50 \mathrm{vol} \%$ (50Ink), and $75 \mathrm{vol} \%$ (75Ink) glycerin solutions all had excellent printing performance, and the printed samples presented regular network structures with distinct layers. There was no deformation or collapse in the high-fidelity printing process. After UV curing, the surfaces of the aerogels appeared misty due to cross-linking. Different from the wet morphology of the samples, the texture and structures of the printed samples with different glycerol content showed a great difference after freeze drying.

As can be clearly seen from Figure $3 a-c$, with the increase of glycerin content, the texture of aerogel gradually changed from white, loose, and porous spongy to translucent gelatinous, and the shape retention ability of dry aerogels were enhanced. After freezedrying, the sample with $75 \%$ glycerin $\left(\mathrm{CNGA}_{75-7}\right)$ could completely keep the regular grid structure formed by 3D printing, while other samples showed different degrees of shrinkage and deformation. 

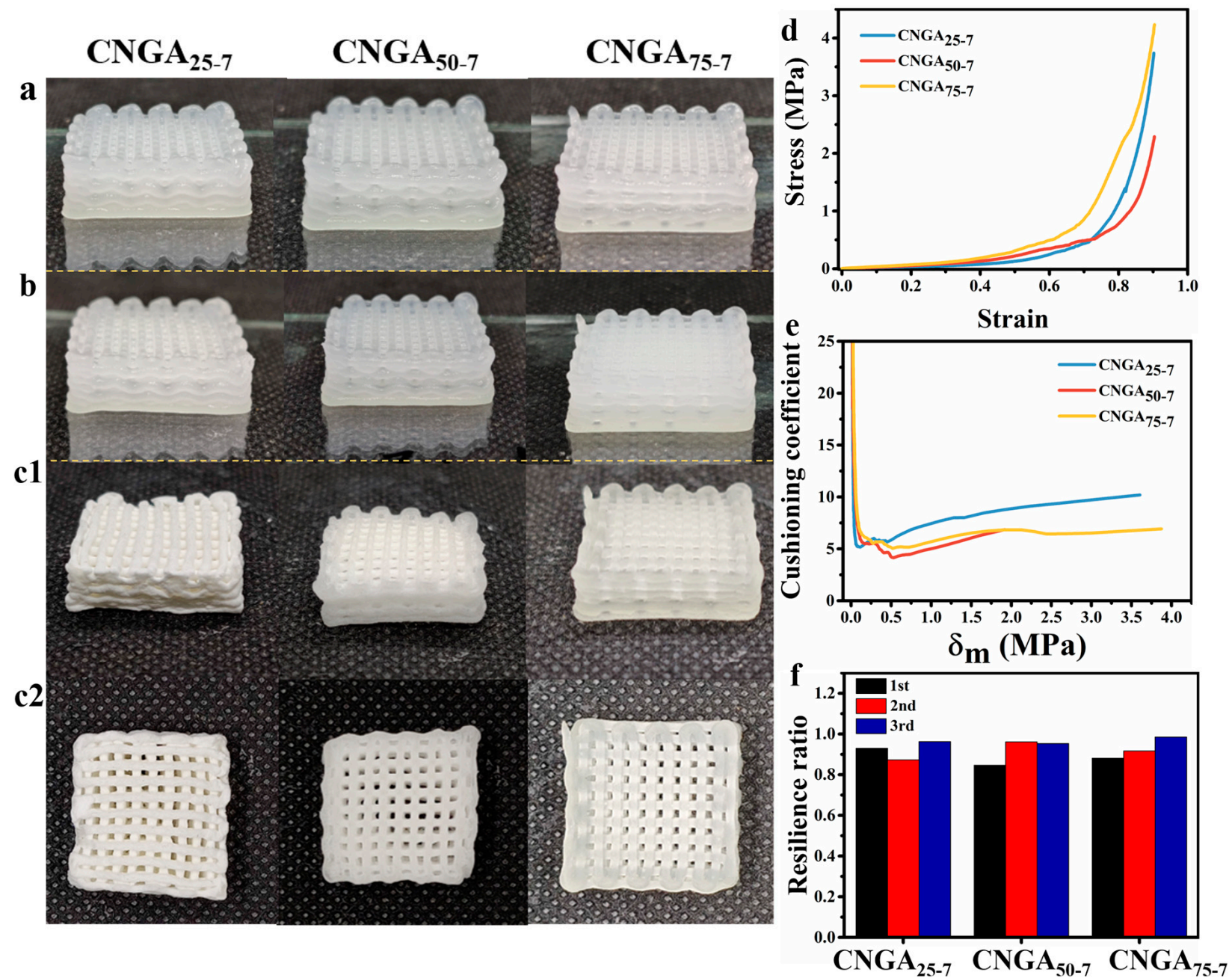

Figure 3. Morphology of 3D-printed samples by CMC-based inks with different glycerin content (a) before and (b) after UV curing and (c1,c2) after freeze drying. (d) Stress-strain curve, (e) cushioning curves, and (f) thrice compression resilience ratio of samples with different glycerin content.

In order to further study the difference of the printed samples with different glycerol content, their microstructures were characterized by SEM. As can be seen from Figure S1a2, the printed sample by $25 \mathrm{Ink}\left(\mathrm{CNGA}_{25-7}\right)$ presented a loose and porous structure with large channels, and the lumps produced by the material fragmentation during the freeze-drying process were attached to its surface. Compared with the $\mathrm{CNGA}_{25-7}$, the $\mathrm{CNGA}_{50-7}$ had a dense structure, but obvious surface wrinkle lines can be observed (Figure S1b2), while the $\mathrm{CNGA}_{75-7}$ had a smooth and denser surface after freeze drying. Therefore, as shown in Figure S1, with the increase of glycerol concentration in the printing ink, the surface micrograph of the obtained samples showed an obvious densification trend, which was conducive to maintaining the shape of the printed samples after freeze drying.

In addition, the differences of static compression performance and elastic recovery energy of samples with different glycerol contents have also been investigated (Figure 3). As can be seen from Figure 3d, with the increase of glycerin content, the elastic modulus and strength of the aerogel gradually increased, indicating that glycerin helped to improve the compression resistance of the printed aerogels. The relationship between the cushioning coefficient of the aerogels and the maximum static stress is shown in Figure 3e. The smaller the cushioning coefficient, the better the cushioning performance of the aerogel [16]. As can be seen, the cushioning properties of $\mathrm{CNGA}_{50-7}$ (4.887) and $\mathrm{CNGA}_{75-7}$ (5.039) are better than those of $\mathrm{CNGA}_{25-7}$ (5.170), indicating that the addition of glycerin improved the cushioning performances of the aerogels. The maximum static pressure corresponding 
to the minimum cushioning coefficient of $\mathrm{CNGA}_{75-7}(0.518 \mathrm{MPa})$ was higher than that of $\mathrm{CNGA}_{50-7}(0.186 \mathrm{MPa})$. Therefore, the application scenarios that match the $\mathrm{CNGA}_{75-7}$ have a wide range of stress options, which is more suitable for the actual storage and transportation of fruits and vegetables. The resilience rate of the aerogels is shown in Figure 3f. The $\mathrm{CNGA}_{25-7}, \mathrm{CNGA}_{50-7}$, and $\mathrm{CNGA}_{75-7}$ samples all had good resilience, and the average resilience of $\mathrm{CNGA}_{75-7}$ was the highest (92.7\%). After comprehensive consideration of the cushioning performance and resilience of the aerogel, 75Ink was selected as the 3D-printing ink for further experiments.

\subsection{Effect of Cross-Linking Time on 3D-Printed Cushioning Aerogels}

Samples with different cross-linking time (before and after UV curing and after lyophilization) are shown in Figure $4 \mathrm{a}-\mathrm{c}$. With the extension of cross-linking time, the degree of cross-linking increased, and the color of the samples showed a tendency to turn yellow after cross-linking and freeze-drying. When the cross-linking time reached $9 \mathrm{~min}$, the printed fiber with network structure shrank, and its diameter became thinner.
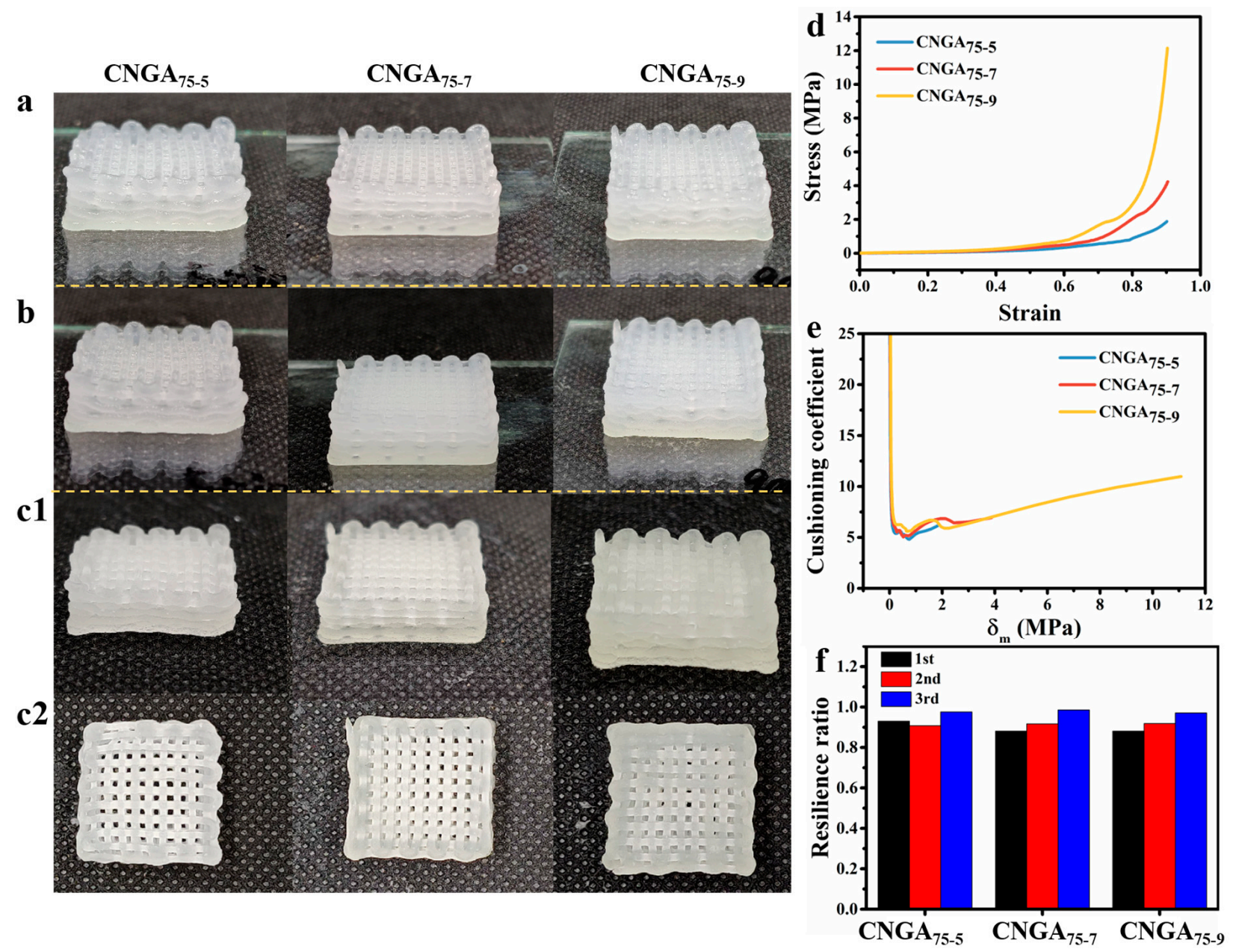

Figure 4. Morphology of 3D-printed samples by 75Ink with different cross-linking time (a) before and (b) after UV curing and (c1,c2) after freeze drying. (d) Stress-strain curve, (e) cushioning curves, and (f) thrice compression resilience ratio of samples with different cross-linking time.

With the increase of cross-linking degree, the elastic modulus of the aerogel increased, and the stress under the same strain also increased with the increase of cross-linking time (Figure 4d). Although the difference of stress between the samples was small in the low strain range, the slope of the stress-strain curve of the aerogel increased sharply when the strain exceeded $60 \%$. In addition, with the extension of cross-linking time, the minimum 
cushioning coefficient of the aerogel presented an increasing trend (Figure 4e). Although the cushioning coefficient of $\mathrm{CNGA}_{75-7}$ is close to that of $\mathrm{CNGA}_{75-5}$, both are lower than that of $\mathrm{CNGA}_{75-9}$. In terms of resilience, the samples with different cross-linking time all had excellent resilience, and the average resilience rates were higher than $90 \%$ (Figure $4 \mathrm{f}$ ). In short, with the increase of cross-linking time, the cushioning performance and resilience of the aerogel both showed a decreasing trend. In addition to the excellent resilience and cushioning performance, the cushioning packaging aerogel should also have the strength of shock resistance. Therefore, the $\mathrm{CNGA}_{75-7}$-printed sample is more suitable to be the fruit cushioning packaging aerogel.

\subsection{Characterization of 3D-Printed Cushioning-Antibacterial Packaging Aerogels}

After successfully obtaining 3D-printed CMC-based cushioning aerogels, chitosan/ AgNPs were immobilized inside the aerogels by coaxial 3D-printing technology to achieve dual functions of cushioning and antibacterial for meeting the practical application requirements. It can be seen that a wide absorption peak in the 400-500 $\mathrm{nm}$ region was observed in the ultraviolet spectrum (Figure 5a), indicating that AgNPs have been successfully synthesized [17]. There were five characteristic peaks in the X-ray diffraction spectrum of AgNPs, located at $38.2^{\circ}, 44.4^{\circ}, 64.6^{\circ}, 77.4^{\circ}$, and $81.6^{\circ}$, corresponding to the (111), (200), (220), (311), and (222) crystal planes of AgNPs, respectively (Figure 5c). This is consistent with the standard diffraction peak of silver nanoparticles (JCPDS no.89-3722). Furthermore, the prepared AgNPs had a uniform particle size, with an average of about $20 \mathrm{~nm}$ (Figure $5 b$ ). As shown in Figure 5d-f, CMC-based 3D-printed cushioning-antibacterial packaging aerogel (CNGA/C-AgNPs) was successfully prepared through coaxial 3D-printing technology. There was a yellow linear region formed by immobilized chitosan/AgNPs in the translucent matrix, and chitosan/AgNPs were embedded in the center of the printed fiber to form a core-shell fiber with a translucent matrix shell and a yellow chitosan/AgNPs core.
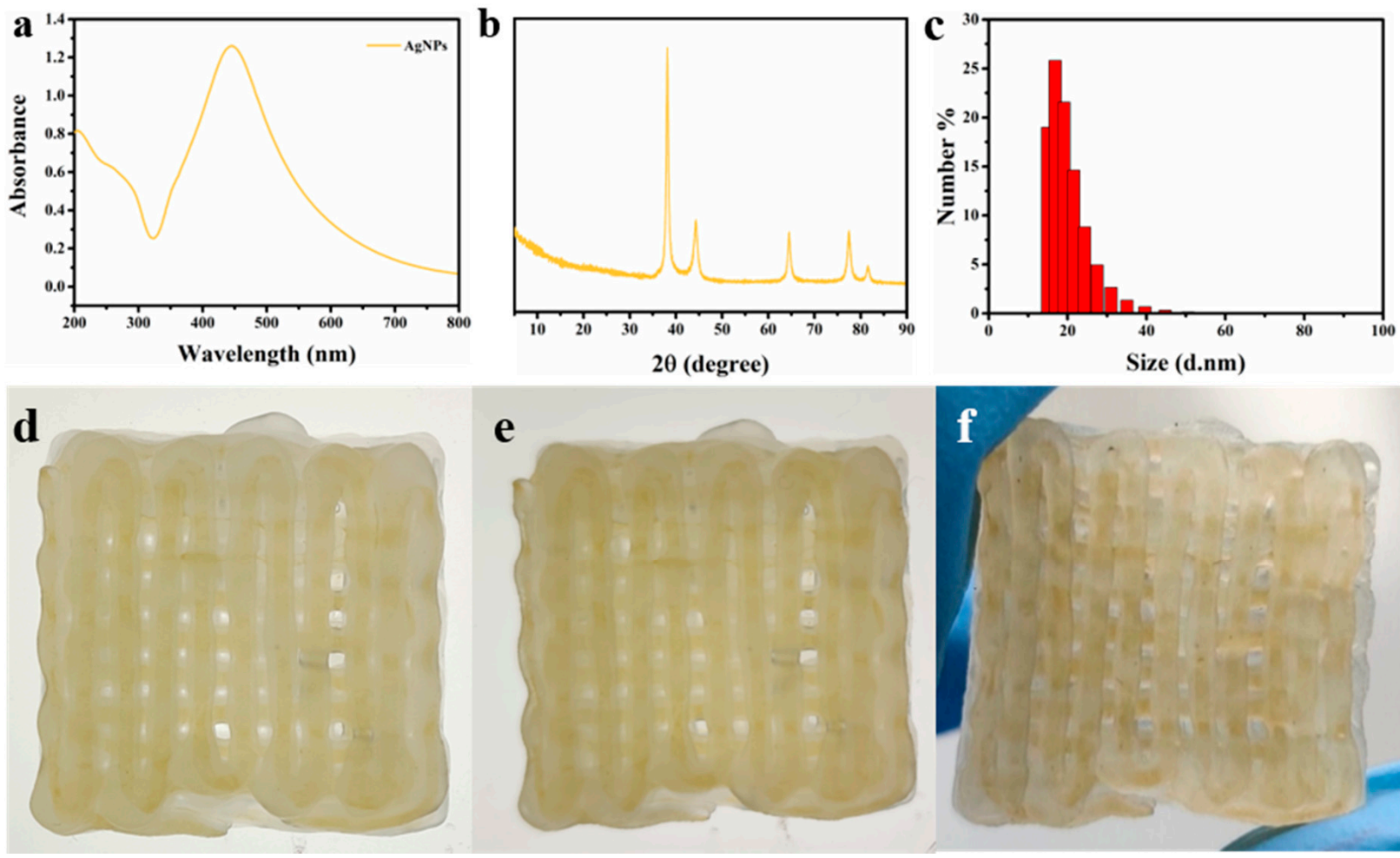

Figure 5. (a) Ultraviolet spectrum, (b) XRD spectra and (c) particle size of silver nanoparticles (AgNPs); photographs of CMC-based 3D-printed aerogel (d) before curing, (e) after curing, and (f) after freeze drying. 
As shown in Figure S2, in the FT-IR spectrum of CMC, the wide peak at $3340 \mathrm{~cm}^{-1}$ was related to $\mathrm{O}-\mathrm{H}$ stretching vibration [18]. The FT-IR characteristic peaks of CMC were the symmetric and asymmetric vibrations of carboxylate at $1601 \mathrm{~cm}^{-1}$ and $1420 \mathrm{~cm}^{-1}$ as well as the C-O-C stretching vibration at $1035-1060 \mathrm{~cm}^{-1}[19,20]$. The characteristic peaks of Cts were at $1640 \mathrm{~cm}^{-1}$ (amide I, C=O stretching vibration) and $1547 \mathrm{~cm}^{-1}$ (amide II, $-\mathrm{NH}_{2}$ bending vibration) [21,22]. The FT-IR characteristic peaks of Cts at $1068 \mathrm{~cm}^{-1}$ and $1030 \mathrm{~cm}^{-1}$ were the stretching vibration peaks of secondary and primary alcohol hydroxyl groups, respectively [23]. The wide band in chitosan (Cts) spectra at 3000-3650 $\mathrm{cm}^{-1}$ was due to the overlap of O-H and N-H stretching vibration. In the FT-IR spectra of $\mathrm{CNGA}_{75-7}$ and CNGA/C-AgNPs, the characteristic peak at $1650 \mathrm{~cm}^{-1}$ was derived from the $\mathrm{C}=\mathrm{O}$ stretching vibration and the $-\mathrm{CH}_{2}$ symmetric stretching in $\mathrm{N}, \mathrm{N}^{\prime}$-methylenebis(acrylamide) and N-(2-hydroxyethyl) acrylamide [24]. The peak at $2925 \mathrm{~cm}^{-1}$ was related to the symmetric stretching of $-\mathrm{CH}_{2}$ in $\mathrm{N}, \mathrm{N}^{\prime}$-methylenebis(acrylamide) [24]. The strong peak at $1033 \mathrm{~cm}^{-1}$ was due to the plane bending vibration of $-\mathrm{OH}$, which may be related to the addition of glycerol [24]. From the above analysis, it can be seen that each component in the aerogels were recombined successfully.

The degradation and swelling properties of packaging aerogels were also investigated (Figure 6). As can be seen from Figure 6a, the degradation rate of CMC-based 3D-printed cushioning-antibacterial packaging aerogels (CNGA/C-AgNPs) in the environment of lysozyme and cellulase reached more than $70 \%$ within 14 days, and the embedding of AgNPs had almost no effect on the degradation rate. In addition, as shown in Figure $6 \mathrm{~b}$, the swelling rate of CNGA/C-AgNPs and $\mathrm{CNGA}_{75-7}$ in $\mathrm{H}_{2} \mathrm{O}$ were $116.67 \%$ and $139.15 \%$, respectively. Meanwhile, those in PBS were $92.31 \%$ and $109.31 \%$, respectively. The swelling rate of the aerogel (CNGA/C-AgNPs) after embedding AgNPs was lower than that before embedding $\left(\mathrm{CNGA}_{75-7}\right)$. This is because the electrostatic interaction between chitosan and carboxymethyl nanocellulose (CMC), and the filling effect of AgNPs in the inner layer of aerogel make it difficult for water to invade. This property is conducive to the moisture stability of the packaging aerogel.

\subsection{The Cushioning Performance of 3D-Printed Cushioning-Antibacterial Packaging Aerogels}

The cushioning performance of CMC-based 3D-printed dual-functional packaging aerogels was evaluated by a universal material testing machine. As shown in Figure $6 c, d$, the minimum cushioning coefficient of the samples decreased from 5.04 to 4.29 after AgNPs were immobilized in the fibers of packaging aerogel. Compared with $\mathrm{CNGA}_{75-7}$, the cushioning performance of CNGA/C-AgNPs was significantly improved. The toughening effect of chitosan/AgNPs and the electrostatic interaction between chitosan and carboxymethyl nanocellulose (CMC) is conducive to the absorption and dissipation of energy. In addition, the cushioning coefficient-maximum static pressure curve of CNGA/CAgNPs sample was similar with that of commercial polyethylene foam (EPE), indicating that they had similar cushioning properties. The average compression resilience ratio of the CNGA/C-AgNPs sample was more than 90\% (Figure 6e), and it had good compression stability (Figure 6f). 
a

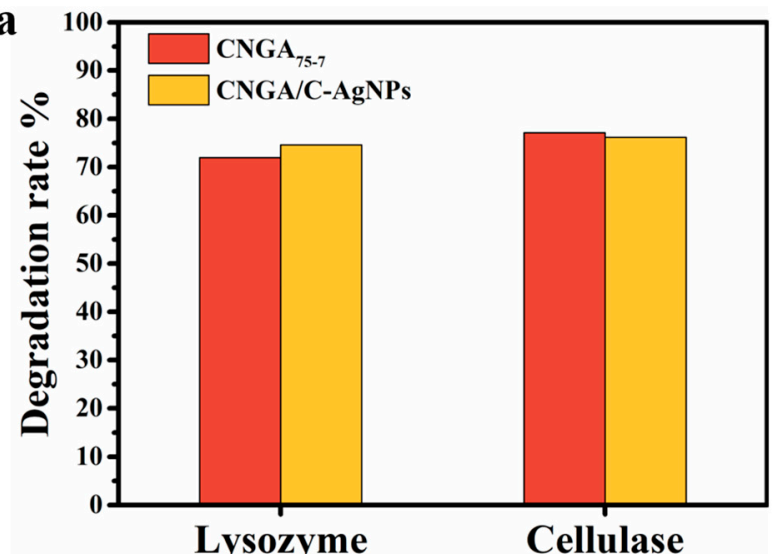

c
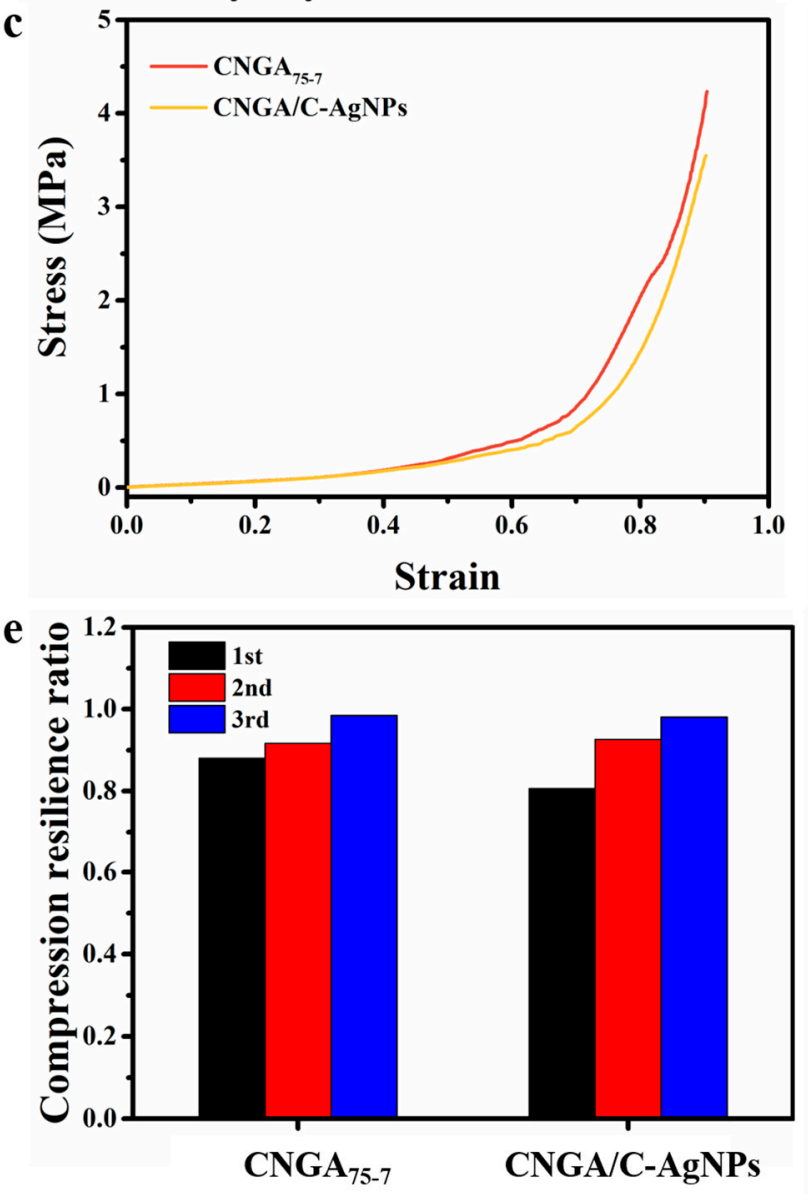

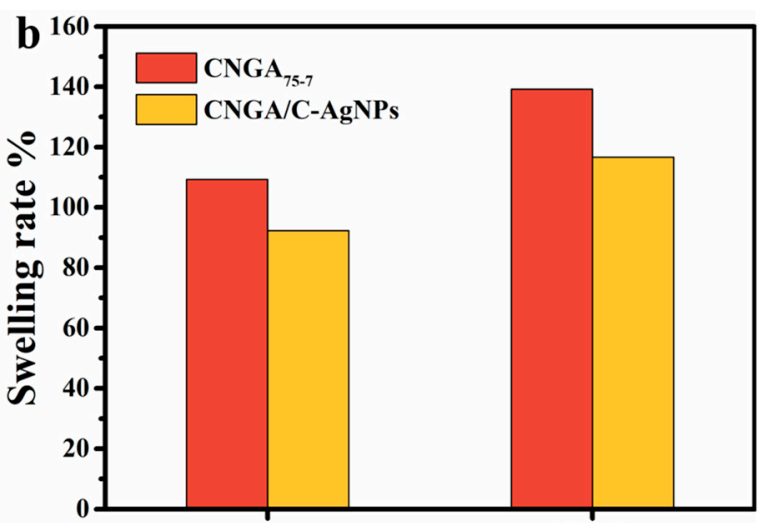

PBS $\quad \mathrm{H}_{2} \mathrm{O}$
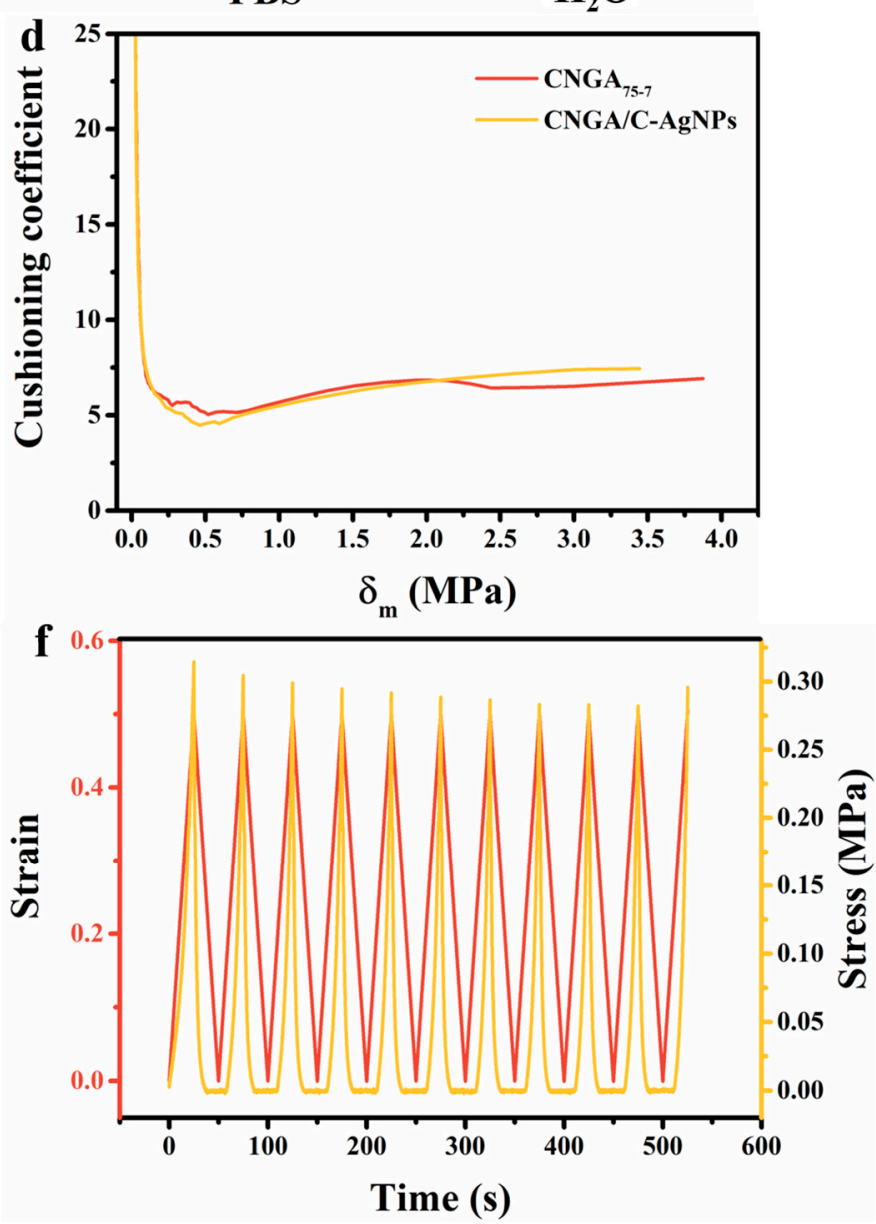

Figure 6. (a) Degradation rate and (b) swelling rate of aerogels before and after immobilizing AgNPs. (c) Stress-strain curve, (d) cushioning curves, and (e) thrice compression resilience ratio of samples with/without AgNPs; (f) compression cycle curve of CNGA/C-AgNPs.

2.6. Ag Release Behavior and Bacterial Inhibition Performance of 3D-Printed Cushioning-Antibacterial Packaging Aerogels

In order to investigate the difference of immobilization effect between the shell core structure formed by coaxial 3D printing and the direct blending printing, the Ag release behavior of the samples obtained by these two printing methods was analyzed (Figure 7a). The shell material (75Ink) and core solution (Chitosan/AgNPs) of CNGA/C-AgNPs were blended to form a new composite, and then, it was used as the printing ink to obtain a CNGA/m-C-AgNPs sample through uniaxial 3D printing. According to Figure 7, in different $\mathrm{pH}$ environments $(\mathrm{pH}=4.0,6.9,9.2)$, the cumulative release of AgNPs of the 
3D-printed cushioning-antibacterial packaging aerogel (CNGA/C-AgNPs) was lower, indicating that the coaxial 3D printing had a good immobilization efficiency for AgNPs, and the packaging aerogel had a good security. It is worth noting that the AgNPs' cumulative release of CNGA/C-AgNPs samples in an acidic environment (Figure $7 \mathrm{~b}$ ) was significantly higher than that in neutral and alkaline environments (Figure $7 \mathrm{c}, \mathrm{d}$ ). This was due to the protonization of chitosan by $\mathrm{H}^{+}$in an acidic environment and the dissolution of the chitosan, which promoted the release of AgNPs. The release rate of the CNGA/C-AgNPs sample tended to be slow after $48 \mathrm{~h}$, while the CNGA/m-C-AgNPs sample showed a long rapid release period of AgNPs, indicating that the immobilization effect of coaxial 3D printing remarkably controlled the release of AgNPs.
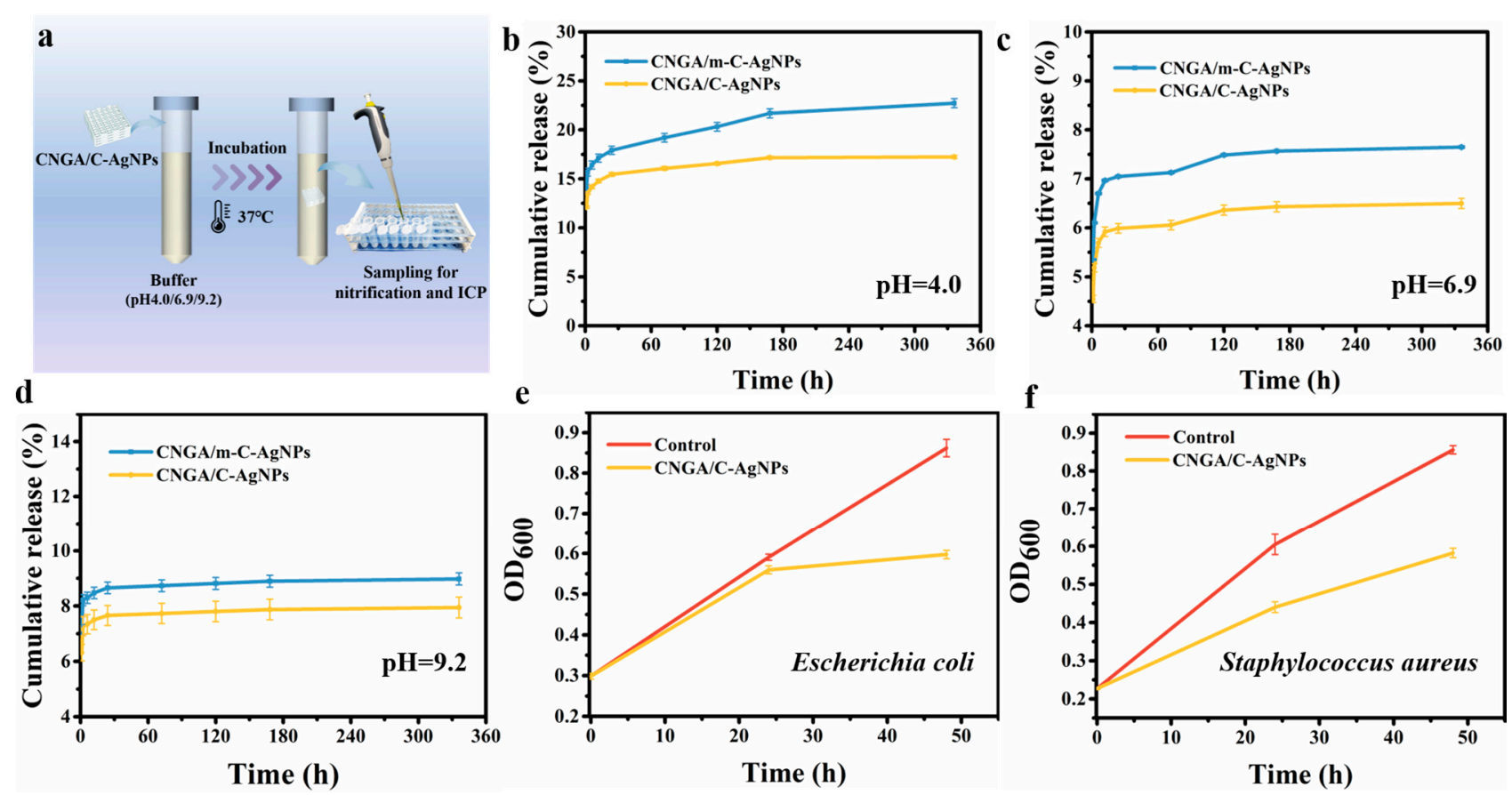

Figure 7. (a) Schematic diagram of Ag release experiment. Cumulative release rates of silver nanoparticles under different $\mathrm{pH}$ : (b) $\mathrm{pH} 4.0$, (c) $\mathrm{pH} 6.9$, and (d) $\mathrm{pH}$ 9.2. $\mathrm{OD}_{600}$ curves of (e) E. coli and (f) S. aureus.

Herein, common foodborne pathogenic bacteria [25], Escherichia coli and Staphylococcus aureus, were selected to evaluate the bacterial inhibition activity of CMC-based 3D-printed cushioning-antibacterial packaging aerogel. In the presence of CNGA/C-AgNPs, the $\mathrm{OD}_{600}$ of bacterial suspension was significantly reduced compared with the control group, indicating that CNGA/C-AgNPs effectively inhibited the proliferation and activity of bacteria. Compared with the control group, with the increase of time, the activity of bacterial inhibition of the material against Escherichia coli increased, and after $24 \mathrm{~h}$, the activity of bacterial inhibition became higher than that in the first $24 \mathrm{~h}$ (Figure 7e). The activity of bacterial inhibition against Staphylococcus aureus had the same trend (Figure 7f). It was because the amount of released antibacterial agents increased with time. In addition, after $24 \mathrm{~h}$, the inhibitory effect of the material on Staphylococcus aureus was better than that of Escherichia coli, which may be related to the structural difference between Gram-negative bacteria and Gram-positive bacteria [26].

\section{Materials and Methods}

\subsection{Materials}

Carboxymethyl nanocellulose (CMC) (carboxyl content: $1.08 \mathrm{mmol} / \mathrm{g}$ ) was purchased from Mujinglin Biotechnology Co., Ltd. (Tianjin, China); chitosan was obtained from Haidebei Marine Biotechnology Co., Ltd. (Jinan, China); tea polyphenol, N, N'methylenebis(acrylamide), and Irgacure 2959 were purchased from MackLin Biochemical 
Technology Co., Ltd. (Shanghai, China); silver nitrate and N-(2-hydroxyethyl) acrylamide were purchased from Aladdin Biochemical Technology Co., Ltd. (Shanghai, China); Escherichia coli and Staphylococcus aureus were provided by Guangzhou Institute of Microbiology (Guangzhou, China). All other reagents were analytical grade.

\subsection{Preparation of Silver Nanoparticles}

First, $0.1 \mathrm{~g}$ of tea polyphenol was dissolved in $10 \mathrm{~mL}$ of deionized water to obtain tea polyphenol solution. Then, $0.34 \mathrm{~g}$ of silver nitrate was dispersed in $45 \mathrm{~mL}$ of deionized water, and then ammonia was added drop by drop until the solution became transparent. Then, $5 \mathrm{~mL}$ of the prepared tea polyphenol solution was added to the silver ammonia solution under stirring, and then, it was reacted at $60^{\circ} \mathrm{C}$ for $1 \mathrm{~h}$ to obtain a silver nanoparticles antimicrobial agent.

\subsection{Preparation of $3 D$-Printing Ink}

Shell ink, $0.2 \mathrm{~g}$ of Irgacure 2959, and $0.32 \mathrm{~g}$ of N, N'-methylenebis(acrylamide) were mixed with $8 \mathrm{~mL}$ of $75 \mathrm{vol} \%$ glycerine solution, respectively, and heated in a water bath at $65^{\circ} \mathrm{C}$ until completely dissolved. Then, $10 \mathrm{~g}$ of carboxymethyl nanocellulose (CMC) were mixed with $4 \mathrm{~mL}$ of Irgacure 2959 solution and $4 \mathrm{~mL}$ of N, $\mathrm{N}^{\prime}$-methylenebis(acrylamide) solution under stirring. Then, $1.2 \mathrm{~g}$ of $\mathrm{N}$-(2-hydroxyethyl) acrylamide was added into the mixture with stirring. The obtained 3D-printing ink was named as 75Ink.

When the $75 \mathrm{vol} \%$ glycerine solution was replaced by the same amount of $50 \mathrm{vol} \%$, $25 \mathrm{vol} \%$ glycerin solution, or DI water, the obtained 3D-printing inks were named as 50Ink, 25Ink, and 0Ink, respectively.

Core solution: $500 \mu \mathrm{L}$ of prepared silver nanoparticles (AgNPs) were dispersed into $5 \mathrm{~mL}$ of $0.5 \mathrm{wt} \%$ chitosan solution containing $1 \%$ acetic acid to be the core solution of coaxial 3D printing.

\subsection{Preparation of 3D-Printed Cushioning Packaging Aerogels}

The 3D-printed CMC-based cushioning packaging aerogel was prepared by Y\&D7300N 3D printer (Yida TEC CO., Dongguan, China) through uniaxial printing technology. Inks with different glycerol content (25Ink, 50Ink, 75Ink) were extruded through $16 \mathrm{G}$ needle $(1.26 \mathrm{~mm})$ to obtain the corresponding samples. The 3D-printed samples were irradiated by UV curing device for $7 \mathrm{~min}$ followed by freeze drying, which were respectively named $\mathrm{CNGA}_{25-7}, \mathrm{CNGA}_{50-7}$, and $\mathrm{CNGA}_{75-7}$.

With the UV curing time modulated for $5 \mathrm{~min}$ and $9 \mathrm{~min}$, the obtained samples were named $\mathrm{CNGA}_{75-5}$ and $\mathrm{CNGA}_{75-9}$, respectively.

\subsection{Preparation of 3D-Printed Cushioning-Antibacterial Packaging Aerogels}

CMC-based cushioning-antibacterial packaging aerogels were prepared by coaxial printing using a Y\&D7300N 3D printer (Yida TEC CO., Donggaun, China). During the printing process, 75Ink was extruded through a coaxial needle $(17 \mathrm{G} / 22 \mathrm{G})$ at a speed of $4 \mathrm{~mm} / \mathrm{s}$. The core solution (Cts/AgNPs) was extruded through an LSP04-1A syringe pump (Longer Pump, Baoding, China) $(3 \mathrm{~mL} / \mathrm{h})$. After printing, UV curing was carried out for $7 \mathrm{~min}$ and then freeze dried to obtain CMC-based cushioning-antibacterial packaging aerogel (CNGA/C-AgNPs).

Then, 75Ink was mixed with the core solution (Cts/AgNPs) to obtain the sample CNGA/m-C-AgNPs through uniaxial 3D printing, which was followed by UV curing and freeze drying. $\mathrm{CNGA} / \mathrm{m}-\mathrm{C}-\mathrm{AgNPs}$ acted as the control group for the Ag release experiment to investigate the influence of the embedding effect of coaxial $3 \mathrm{D}$ printing on the release behavior of AgNPs.

\subsection{Rheological Test of 3D-Printing Inks}

The rheological test was carried out by a ARES-G2 rheometer (TA Instruments, New Castle, DE, USA) equipped with $40 \mathrm{~mm}$ parallel plates. 
Flow Sweep: The flow sweep measurements were conducted at the shear rate range of $10^{-2}-10^{2} \mathrm{~s}^{-1}$ at $25^{\circ} \mathrm{C}, 5$ points per decade. The samples reach the equilibrium temperature for $180 \mathrm{~s}$ prior to the measurement.

Oscillation Amplitude: The test was conducted at stain from $0.01 \%$ to $100 \%$ with angular frequency of $6.28 \mathrm{rad} / \mathrm{s}$ at $25^{\circ} \mathrm{C}$.

\subsection{Characterization of 3D-Printed Packaging Aerogels}

UV-vis spectra were measured by UV-1800 (Shimazu, Kyoto, Japan). The particle size distribution was measured using the SZ-100Z Nanoparticle Analyzer (Horiba, Kyoto, Japan). Scanning electron microscope (SEM) micrographs were obtained by a Merlin (Zeiss, Germany) microscope. FT-IR spectrometer (VERTEX 70, Bruker, Karlsruhe, Germany) recorded from 4000 to $400 \mathrm{~cm}^{-1}$ and X-ray diffraction spectrometer (X'pert Powder, PANalytical, Almelo, Netherlands) worked with $\mathrm{Cu} \mathrm{K} \alpha$ radiation in the scan range of $2 \theta$ from $5^{\circ}$ to $90^{\circ}$.

\subsection{Cushioning and Resilience Performance of 3D-Printed Packaging Aerogels}

The cushioning and resilience performance of the samples were measured by an INSTRON 5565 electronic universal material tester (Instron, Boston, MA, USA).

Cushioning performance: The data collected in the static compression experiment (compression rate: $5 \mathrm{~mm} / \mathrm{min}$, final strain: 90\%) were calculated according to the following formulas to evaluate the cushioning performance of the aerogels. The smaller the cushioning coefficient $C$, the more energy is absorbed per unit volume of the materials and the higher the cushioning efficiency. The minimum value of the cushioning factor usually corresponds to the best application scenario of the material.

$$
\begin{gathered}
e=\int_{0}^{\varepsilon} \delta d \varepsilon \\
\mathrm{C}=\frac{\delta}{e}
\end{gathered}
$$

Among them, $\delta$ is the compressive stress of the sample, and $\varepsilon$ is the compressive strain of the sample.

Resilience performance: The test was carried out as described by Li et al. [27] with slight modification. The loading speed during the compression process was $12 \mathrm{~mm} / \mathrm{min}$. Afterwards, the samples were compressed to 50\% strain and held for $3 \mathrm{~min}$. Then, the thickness of the samples was measured after $10 \mathrm{~s}$ of recovery. The compression test was repeated three times for each sample. The resilience rate of the sample was calculated according to the following formula.

$$
R_{j}=\frac{t_{j}-\left(\frac{T_{i}}{2}\right)}{\frac{T_{i}}{2}}
$$

where $R_{j}$ is the resilience rate of the $j$ th time, $j=1,2,3 ; T_{j}$ and $T_{j}$ are respectively the thickness before and after the $j$ th compression of the samples, $j=1,2,3$.

\subsection{Biodegradation and Swelling Performance of the 3D-Printed Packaging Aerogels}

Biodegradation performance: A certain mass $(0.2 \mathrm{~g})$ of sample was immersed in $10 \mathrm{~mL}$ PBS containing $2 \times 10^{4} \mathrm{U} / \mathrm{mL}$ lysozyme or $150 \mathrm{U} / \mathrm{mL}$ cellulase, respectively, incubated at $37^{\circ} \mathrm{C}$ for 14 days, and then taken out, freeze dried, and weighed. The degradation rate of sample $(D)$ was calculated by the following formula:

$$
D \%=\frac{W_{0}-W_{t}}{W_{0}} \times 100 \%
$$


where $W_{0}(\mathrm{~g})$ is the initial sample weight, and $W_{t}(\mathrm{~g})$ is the sample weight after 14 days.

Swelling performance: The swelling performance of the aerogels was measured by the following method: The aerogels were immersed in PBS and deionized water respectively, and taken out after keeping at $25^{\circ} \mathrm{C}$ for $24 \mathrm{~h}$. The liquid on the surface was dried with a filter paper and weighed. The swelling rate (S) can be calculated according to the following formula:

$$
\mathrm{S} \%=\frac{\mathrm{m}_{1}-\mathrm{m}_{0}}{\mathrm{~m}_{0}} \times 100 \%
$$

where $\mathrm{m}_{0}(\mathrm{~g})$ is initial sample weight, and $\mathrm{m}_{1}(\mathrm{~g})$ is the sample weight after swelling for $24 \mathrm{~h}$.

\subsection{The AgNPs Release Behavior of 3D-Printed Packaging Aerogels}

The 3D-printed CMC-based cushioning-antibacterial packaging aerogel was immersed in $10 \mathrm{~mL}$ of buffer solution $(\mathrm{pH}=4.0,6.9,9.2)$ and incubated at $37^{\circ} \mathrm{C}$, which was followed by taking out $0.5 \mathrm{~mL}$ of sample solution and adding the same amount of fresh buffer at specific times $(t=1,2,6,12,24,72,120,168,336 \mathrm{~h})$. Then, $4.5 \mathrm{~mL}$ of nitric acid solution (1M) was added to the sample solution for nitrification, and the silver content was detected by PE 8300 inductively coupled plasma emission spectrometer (PerkinElmer, Waltham, MA, USA).

\subsection{Bacterial Inhibition Activity of 3D-Printed Packaging Aerogels}

The bacterial inhibition activity of 3D-printed CMC-based cushioning-antibacterial packaging aerogel was evaluated by the following methods: Firstly, the colonies of Escherichia coli and Staphylococcus aureus were scraped and dispersed in the LB liquid medium to prepare the bacterial suspension of Escherichia coli and Staphylococcus aureus, respectively. Certain mass samples $(0.2 \mathrm{mg})$ were soaked in the bacterial suspension and cultured at $37^{\circ} \mathrm{C}$. Then, the bacterial suspension was taken out at specific times $(0,12,24 \mathrm{~h})$ for measuring the $\mathrm{OD}_{600}$ value by Multiskan Go enzyme spectrometer (Thermo Scientific, Waltham, MA, USA). The bacterial inhibition activity of the samples was analyzed by the difference of $\mathrm{OD}_{600}$ value between the experimental group and the control group.

\section{Conclusions}

In this study, CMC-based cushioning-antimicrobial dual-function food packaging aerogel (CNGA/C-AgNPs) was successfully prepared by using carboxymethyl nanocellulose as the matrix, glycerol/acrylamide derivative as supplemented, and AgNPs were immobilized as the core layer through coaxial 3D-printing technology. The prepared $\mathrm{CNGA} / \mathrm{C}-\mathrm{AgNPs}$ packaging aerogel has good cushioning and resilience performance. The hollow structure constructed by coaxial 3D-printing technology has a good immobilization efficiency for AgNPs. Additionally, the packaging aerogel has an obvious bacterial inhibition effect on E. coli and S. aureus. Meanwhile, the packaging aerogel with cushioningantimicrobial dual function is biodegradable. Hence, coaxial 3D-printing technology has great application potential in the construction of food packaging aerogels, especially cushioning packaging for the storage and transportation of fruits and vegetables.

Supplementary Materials: The following are available online, Figure S1: Microscopic morphology of (a1, a2) $\mathrm{CNGA}_{25-7}$; (b1, b2) $\mathrm{CNGA}_{50-7}$; $(\mathrm{c} 1, \mathrm{c} 2) \mathrm{CNGA}_{75-7}$ after freeze-drying, Figure S2: FT-IR spectra of CMC, Cts, $\mathrm{CNGA}_{75-7}$ and CNGA/C-AgNPs.

Author Contributions: Conceptualization, W.Z.; Investigation, W.Z., J.F. and S.T.; Methodology, W.Z. and J.F.; Project administration, Z.W.; Supervision, Z.W. and X.W.; Visualization, W.Z., J.F. and S.T.; Writing-original draft, W.Z. and J.F.; Writing—review and editing, Z.W. and X.W. All authors have read and agreed to the published version of the manuscript.

Funding: This research was funded by the Guangdong Basic and Applied Basic Research Foundation (No. 2020A1515110004), the China Postdoctoral Science Foundation (No. 2020M682716), and Science and Technology Planning Project of Guangzhou City (No. 202102020007). 
Institutional Review Board Statement: Not applicable.

Informed Consent Statement: Not applicable.

Data Availability Statement: Not applicable.

Acknowledgments: In this section, you can acknowledge any support given which is not covered by the author contribution or funding sections. This may include administrative and technical support, or donations in kind (e.g., materials used for experiments).

Conflicts of Interest: The authors declare no conflict of interest.

Sample Availability: Samples of the compounds are not available from the authors.

\section{References}

1. Lin, M.H.; Chen, J.H.; Chen, F.; Zhu, C.Q.; Wu, D.; Wang, J.; Chen, K.S. Effects of cushioning materials and temperature on quality damage of ripe peaches according to the vibration test. Food Packag. Shelf Life 2020, 25, 100518. [CrossRef]

2. Jarimopas, B.; Singh, S.P.; Sayasoonthorn, S.; Singh, J. Comparison of package cushioning materials to protect post-harvest impact damage to apples. Packag. Technol. Sci. 2007, 20, 315-324. [CrossRef]

3. Zhou, R.; Su, S.Q.; Li, Y.F. Effects of cushioning materials on the firmness of Huanghua pears (Pyrus pyrifolia Nakai cv. Huanghua) during distribution and storage. Packag. Technol. Sci. 2008, 21, 1-11. [CrossRef]

4. Guo, A.; Zhao, J.; Li, J.; Li, F.; Guan, K. Forming parameters optimisation of biomass cushion packaging material by orthogonal test. Mater. Res. Innov. 2015, 19, 521-525. [CrossRef]

5. De France, K.J.; Hoare, T.; Cranston, E.D. Review of Hydrogels and Aerogels Containing Nanocellulose. Chem. Mater. 2017, 29, 4609-4631. [CrossRef]

6. Moon, R.J.; Martini, A.; Nairn, J.; Simonsen, J.; Youngblood, J. Cellulose nanomaterials review: Structure, properties and nanocomposites. Chem. Soc. Rev. 2011, 40, 3941-3994. [CrossRef]

7. Hettrich, K.; Pinnow, M.; Volkert, B.; Passauer, L.; Fischer, S. Novel aspects of nanocellulose. Cellulose 2014, 21, 2479-2488. [CrossRef]

8. Kalia, A.; Parshad, V.R. Novel Trends to Revolutionize Preservation and Packaging of Fruits/Fruit Products: Microbiological and Nanotechnological Perspectives. Crit. Rev. Food Sci. Nutr. 2015, 55, 159-182. [CrossRef] [PubMed]

9. Campos, C.A.; Gerschenson, L.N.; Flores, S.K. Development of Edible Films and Coatings with Antimicrobial Activity. Food Bioprocess Technol. 2011, 4, 849-875. [CrossRef]

10. Hartemann, P.; Hoet, P.; Proykova, A.; Fernandes, T.; Baun, A.; De Jong, W.; Filser, J.; Hensten, A.; Kneuer, C.; Maillard, J.-Y.; et al. Nanosilver: Safety, health and environmental effects and role in antimicrobial resistance. Mater. Today 2015, 18, 122-123. [CrossRef]

11. Li, L.; Zhao, C.; Zhang, Y.; Yao, J.; Yang, W.; Hu, Q.; Wang, C.; Cao, C. Effect of stable antimicrobial nano-silver packaging on inhibiting mildew and in storage of rice. Food Chem. 2017, 215, 477-482. [CrossRef]

12. Sarwar, M.S.; Niazi, M.B.K.; Jahan, Z.; Ahmad, T.; Hussain, A. Preparation and characterization of PVA/nanocellulose/Ag nanocomposite films for antimicrobial food packaging. Carbohydr. Polym. 2018, 184, 453-464. [CrossRef]

13. Wang, Z.; Xia, T.; Liu, S. Mechanisms of nanosilver-induced toxicological effects: More attention should be paid to its sublethal effects. Nanoscale 2015, 7, 7470-7481. [CrossRef]

14. Braakhuis, H.M.; Gosens, I.; Krystek, P.; Boere, J.A.F.; Cassee, F.R.; Fokkens, P.H.B.; Post, J.A.; van Loveren, H.; Park, M.V.D.Z Particle size dependent deposition and pulmonary inflammation after short-term inhalation of silver nanoparticles. Part. Fibre Toxicol. 2014, 11, 49. [CrossRef]

15. Wang, X.; Jiang, M.; Zhou, Z.; Gou, J.; Hui, D. 3D printing of polymer matrix composites: A review and prospective. Compos. Part $B$ Eng. 2017, 110, 442-458. [CrossRef]

16. Luo, Y.Y.; Xiao, S.L.; Li, S.L. Effect of Initial Water Content on Foaming Quality and Mechanical Properties of Plant Fiber Porous Cushioning Materials. Bioresources 2017, 12, 4259-4269. [CrossRef]

17. Ceballos, R.L.; von Bilderling, C.; Guz, L.; Bernal, C.; Fama, L. Effect of greenly synthetized silver nanoparticles on the properties of active starch films obtained by extrusion and compression molding. Carbohydr. Polym. 2021, 261, 117871. [CrossRef] [PubMed]

18. Zhang, D.; Liu, H.; Chen, M.; Wang, Q.; Feng, J.; Shu, X.; Li, C.; Li, Y.; Xie, X.; Shi, Q. A series of carboxymethyl cellulose-based antimicrobial peptide mimics were synthesized for antimicrobial applications. Carbohydr. Polym. 2021, 261, 117822. [CrossRef] [PubMed]

19. Lakshmi, D.S.; Trivedi, N.; Reddy, C.R.K. Synthesis and characterization of seaweed cellulose derived carboxymethyl cellulose. Carbohydr. Polym. 2017, 157, 1604-1610. [CrossRef]

20. Ni, W.; Zheng, Z.; Liu, H.; Wang, P.; Wang, L.; Wang, H.; Sun, X.; Yang, Q.; Tang, H.; Zhao, G. Synthesis of the carboxymethyl cellulose magnetic nanoparticles for efficient immobilization of prenyltransferase NovQ. Carbohydr. Polym. 2020, $235,115955$. [CrossRef] 
21. Wang, H.; Liu, H.; Chu, C.; She, Y.; Jiang, S.; Zhai, L.; Jiang, S.; Li, X. Diffusion and Antibacterial Properties of Nisin-Loaded Chitosan/Poly (L-Lactic Acid) Towards Development of Active Food Packaging Film. Food Bioprocess Technol. 2015, 8, $1657-1667$. [CrossRef]

22. Theapsak, S.; Watthanaphanit, A.; Rujiravanit, R. Preparation of Chitosan-Coated Polyethylene Packaging Films by DBD Plasma Treatment. ACS Appl. Mater. Interfaces 2012, 4, 2474-2482. [CrossRef]

23. Wang, W.; Hu, J.; Zhang, R.; Yan, C.; Cui, L.; Zhu, J. A pH-responsive carboxymethyl cellulose/chitosan hydrogel for adsorption and desorption of anionic and cationic dyes. Cellulose 2021, 28, 897-909. [CrossRef]

24. Lalita; Singh, A.P.; Sharma, R.K.R. Selective sorption of Fe(II) ions over Cu(II) and Cr(VI) ions by cross-linked graft copolymers of chitosan with acrylic acid and binary vinyl monomer mixtures. Int. J. Biol. Macromol. 2017, 105, 1202-1212. [CrossRef]

25. De Oliveira Vieira, K.C.; da Silva, H.R.A.; Rocha, I.P.M.; Barboza, E.; Eller, L.K.W. Foodborne pathogens in the omics era. Crit. Rev. Food Sci. Nutr. 2021, 62,1-16. [CrossRef]

26. Siripatrawan, U.; Vitchayakitti, W. Improving functional properties of chitosan films as active food packaging by incorporating with propolis. Food Hydrocoll. 2016, 61, 695-702. [CrossRef]

27. Li, F.Y.; Guan, K.K.; Liu, P.; Li, G.; Li, J.F. Ingredient of Biomass Packaging Material and Compare Study on Cushion Properties. Int. J. Polym. Sci. 2014, 2014, 146509. [CrossRef] 